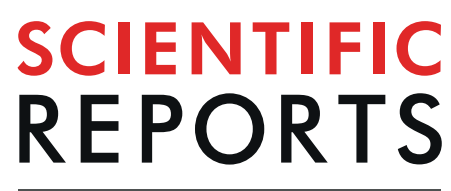

natureresearch

\title{
Lineage tracing reveals the hierarchical relationship between neural stem cell populations in the mouse forebrain
}

\author{
Nadia Sachewsky ${ }^{1,6}$, Wenjun $X_{u^{1,2,6}}$, Tobias Fuehrmann $\mathbb{1}^{4}$, Derek van der Kooy ${ }^{2,3} \&$ \\ Cindi M. Morshead ${ }^{1,2,4,5 *}$
}

Since the original isolation of neural stem cells (NSCs) in the adult mammalian brain, further work has revealed a heterogeneity in the NSC pool. Our previous work characterized a distinct, Oct4 expressing, NSC population in the periventricular region, through development and into adulthood. We hypothesized that this population is upstream in lineage to the more abundant, well documented, GFAP expressing NSC. Herein, we show that Oct4 expressing NSCs give rise to neurons, astrocytes and oligodendrocytes throughout the developing brain. Further, transgenic inducible mouse models demonstrate that the rare Oct4 expressing NSCs undergo asymmetric divisions to give rise to GFAP expressing NSCs in naïve and injured brains. This lineage relationship between distinct NSC pools contributes significantly to an understanding of neural development, the NSC lineage in vivo and has implications for neural repair.

The adult mammalian central nervous system contains two distinct populations of neural stem cells (NSCs). Within the well-documented neurogenic niche of the adult forebrain germinal zone, glial fibrillary acidic protein (GFAP) expressing definitive NSCs (dNSC) infrequently divide with a cell cycle time of 2-4 weeks to produce more rapidly dividing progenitors that migrate along the rostral migratory stream towards the olfactory bulb and generate interneurons $\mathrm{s}^{1-4}$. A second population of primitive NSCs (pNSCs), initially isolated during development as early as embryonic day (E) 5.5, persists into adulthood. The pNSC does not express GFAP, but does express low levels of the pluripotency marker, Oct $4^{5-7}$. Primitive NSCs are rare (more than 10 fold less frequent than dNSCs) and have a long cell cycle time of 3-5 months in the adult brain ${ }^{4,7}$.

In vitro, dNSCs proliferate in the presence of epidermal growth factor (EGF) and fibroblast growth factor (FGF) to form clonally derived colonies of cells termed neurospheres ${ }^{8}$. pNSCs generate clonally derived, multipotent colonies in the presence of leukemia inhibitory factor (LIF) ${ }^{6,7}$. Definitive and primitive NSCs can be further differentiated based on the expression of cell surface markers c-kit and ErbB2 ${ }^{9}$. Previous work supports the hypothesis that pNSCs are lineally related to dNSCs ${ }^{7}$. First, in vitro culture experiments demonstrate that adult-derived pNSC colonies can be induced to form dNSC neurospheres (in EGF + FGF + heparin, EFH) but not vice versa, and second, transplantation of pNSCs onto the rostral migratory stream (RMS) revealed pNSC derived neuroblasts on the RMS and neurons in the olfactory bulb. Further, Reeve et al. ${ }^{4}$ showed that in conditional Oct4 loss of function mice (Oct4fl/f;;Sox1Cre), repopulation of the dNSC population following ablation does not occur. These findings support the hierarchical lineage relationship between pNSCs and dNSCs.

Herein we have used several transgenic mouse models that permit lineage tracking of pNSC progeny in embryonic, neonatal and adult mice. Using labeling paradigms in the developing CNS, in combination with in vivo ablation paradigms in the adult, we demonstrate that pNSCs are the precursors to dNSCs in vivo. Retroviral lineage tracing and a triple transgenic mouse (Oct4CreER $\left.{ }^{\mathrm{T} 2} ; \mathrm{ROSAyfp}^{\mathrm{f} / \mathrm{f}} ; \mathrm{GFAPtk}\right)$ that permits pre-labeling of the

\footnotetext{
${ }^{1}$ Department of Surgery, Division of Anatomy, University of Toronto, Ontario, Canada. ${ }^{2}$ Institute of Medical Sciences, University of Toronto, Ontario, Canada. ${ }^{3}$ Department of Molecular Genetics, University of Toronto, Ontario, Canada. ${ }^{4}$ Institute of Biomaterials and Biomedical Engineering, University of Toronto, Ontario, Canada. ${ }^{5}$ Toronto Rehabilitation Institute, University Health Network, Toronto, Ontario, Canada. ${ }^{6}$ These authors contributed equally: Nadia Sachewsky and Wenjun Xu. *email: cindi.morshead@utoronto.ca
} 
very rare Oct $4+$ cells in vivo. We show that Oct $4+$ pNSCs can give rise to dNSCs in the adult mouse periventricular region in naïve brains and in a loss of function model of dNSC repopulation.

\section{Results}

Embryonic pNSCs give rise to dNSCs and all neural cell types in the brain. Primitive NSCs express the pluripotency marker Oct4, which distinguishes this population from $\mathrm{dNSCs}^{7}$. To determine whether pNSCs give rise to dNSCs in vivo, we took advantage of an inducible Oct4CreER ${ }^{\mathrm{T} 2}$ crossed to a reporter mouse $\left(\right.$ ROSAyfp $^{\mathrm{f} / / \mathrm{fl}}$ or ROSA26-tdtomato ${ }^{\mathrm{f} / \mathrm{fl}}$ ) to enable the selective labeling of pNSCs. Using the transgenic reporter line Oct4CreER ${ }^{\mathrm{T} 2}$; ROSA26-tdtomato ${ }^{\mathrm{f} / \mathrm{fl}}$, we labeled the pNSCs when they are most abundant in the developing brain ${ }^{7}$. Timed pregnant females were injected with tamoxifen once a day at gestational age E16-18, a period when the developing brain is expanding the size of the neural precursor pool and undergoing neurogenesis and gliogene$\operatorname{sis}^{10-12}$ (Fig. 1a). We predicted that pNSCs labeled in the Oct4CreER ${ }^{\mathrm{T} 2}$; ROSA26-tdtomato ${ }^{\mathrm{f} / \mathrm{fl}}$ mice would generate progeny of all three lineages as well as give rise to dNSCs.

Consistent with the prediction that the pNSC lies at the top of the neural cell lineage, we found tdTomato+ cells, derived from an Oct4 + pNSC, in all areas of the brain at PND21 (Fig. 1b). tdTomato+ cells were found in the germinal zone of the lateral ventricle (Fig. 1bi) where neural precursor cells are found, as well as in the olfactory bulb (Fig. 1bii), the cortex (Fig. 1biv), and striatum (Fig. 1bv). The tdTomato + cells had the morphology of neurons and subpopulations of cells were co-labeled with glial markers (GFAP and MBP; astrocytes and oligodendrocytes, respectively) (Fig. 1biii,vi). To confirm the derivation of other lineages from pNSC derived cells, high magnification, confocal images confirmed the co-expression of tdTomato + , Oct 4 expressing pNSC derived cells of mature phenotypes including oligodendrocytes (Olig2), neurons (NeuN), and astrocytes (GFAP) as well as uncommitted progenitors (Sox2)(Fig. 1c). We found tdTomato + cells in all Oct4CreER ${ }^{\mathrm{T} 2}$; ROSA26-tdtomato ${ }^{\mathrm{f} /}$ ${ }^{\mathrm{fl}}$ that received TAM (4/4). The tfTomato+ cells differentiated into all lineages examined (Fig. 1d). Interestingly, the bias for the distribution of tdTomato + cells is towards the germinal zone at this time point, consistent with the idea that these cells are derived from pNSCs (Fig. 1e). Hence, during embryogenesis, rare, Oct4+ pNSCs contribute to neural cells throughout the developing brain.

A separate cohort of Oct4CreER ${ }^{\mathrm{T} 2}$; ROSA26-tdtomato ${ }^{\mathrm{f} / \mathrm{fl}}$ mice received TAM through the mother's milk to label pNSCs in the early postnatal brain. The mothers were injected with TAM on PND2-4 (Fig. 1f), and the mice were sacrificed at PND14 and processed for the pNSC-derived, tdTomato+ LIF colonies; a time when we have observed significantly more LIF colonies compared to adult mice. Consistent with our predictions, we observed tdTomato + LIF colonies from all mice examined $(n=8)(4.625 \pm 0.73$ tdTomato + LIF colonies/mouse) (Fig. 1g).

Based on these findings in the early postnatal brain, we predicted that labeling pNSCs in Oct4CreER ${ }^{\mathrm{T} 2}$;ROSAyfp ${ }^{\mathrm{fl} / \mathrm{fl}}$ mice would result in a cohort of YFP $+\mathrm{dNSCs}$ which could be identified in the neurosphere assay in adult mice. Postnatal day 1-2 (PND1-2) mice were exposed to TAM through the mother's milk for 14 days to maximize the labeling of a cohort of Oct4 + pNSCs, and the neurosphere assay was performed in young adult mice (Fig. 1h). As predicted, YFP + pNSC-derived colonies were isolated from adult mice (mean $=6.46 \%$ of all LIF responsive colonies) (Fig. 1i). We also observed a cohort of YFP+ dNSC-derived neurospheres (mean $=13.87 \%$ ), indicating that dNSCs were derived from Oct4+ cells labeled in the early postnatal period (Fig. 1j). Most important, no YFP+ neurospheres were derived from cultures of TAM fed Oct4CreER ${ }^{\mathrm{T} 2-1-} ; \mathrm{ROSAyfp}^{\mathrm{f} / \mathrm{fl}}$ littermate controls and non-fed Oct4CreER ${ }^{\mathrm{T} 2} ; \mathrm{ROSAyfp}^{\mathrm{f} / / \mathrm{l}}$ indicating promoter-specific labeling. A separate cohort of TAM fed Oct $4 \mathrm{CreER}^{\mathrm{T} 2}$; ROSA26-tdtomato ${ }^{\mathrm{f} / \mathrm{fl}}$ mice were perfused and wholemount preparations the lateral ventricles revealed the presence of Tdtomato + cells in young adult mice (Fig. 1k). Hence, Oct4+ pNSCs can give rise to dNSC progeny in vivo, under non-injury conditions.

Taken together, these data demonstrate the lineage relationship between pNSCs and dNSCs under baseline conditions, consistent with work done previously ${ }^{7}$. We next asked whether this lineage relationship persisted into the adult brain when the pNSCs primarily are quiescent in vivo but are activated in response to injury $y^{4,7}$.

dNSCs are repopulated by GFAP negative cells. Previous studies have shown that the administration of ganciclovir (GCV), with or without the mitotic inhibitor AraC, to GFAPtk mice results in a complete loss of dNSC-derived neurospheres within a few days of treatment ${ }^{3,7}$. However, the AraC and GCV ablation paradigm does not result in a permanent depletion of dNSCs and over time, dNSC repopulation occurs ${ }^{7}$. We postulated that the GFAP negative (pNSC), or a quiescent dNSC (GFAP + ), contributed to this repopulation. To address this question we administered tamoxifen (TAM) for 2 weeks to young adult triple transgenic GFAPCreER $^{\mathrm{T} 2}$;ROSAyfp ${ }^{\mathrm{t} / \mathrm{fl}}$;GFAPtk mice (herein termed GFAPCRE +/tk) to label a cohort of GFAP + dNSCs (Fig. 2a). This labeling paradigm resulted in $32-56 \%$ of the dNSCs derived clonal neurospheres expressing YFP in both the experimental strain (GFAPCRE $+/ \mathrm{tk}+$ ) and the littermate control strain (GFAPCRE $+/ \mathrm{tk}-$ ).

After establishing baseline labeling, dNSC ablation was performed using AraC and GCV. Mice received 7 days of intraventricular AraC infusion followed by 3 days of intraventricular GCV to selectively and completely ablate dividing GFAP + cells, as previously described ${ }^{7}$ (Fig. 2a). Immediately following ablation (day 10), there was a complete loss of dNSC-derived, EFH neurospheres from GFAPCRE $+/$ tk + mice (Fig. 2bi). Control mice (GFAPCRE +/tk -$)$ had a YFP + cohort $(28 \pm 11 \%$ of the total clonal neurospheres formed $)$ that was not killed by the GCV due to the lack of the tk transgene (Fig. 2bi). As predicted, none of the pNSC, LIF responsive clonal colonies expressed YFP confirming that pNSCs are GFAP negative (Fig. 2ci). At 14- and 30-days post ablation (day 24 and day 40, respectively), the dNSC pool expanded and repopulated the subependyma, as indicated by the increase in total EFH clonal neurosphere numbers. Notably, none of the GFAPCRE $+/$ tk $+\mathrm{dNSC}$-derived neurospheres expressed YFP, revealing that the dNSC-derived neurospheres did not originate from the previously labeled GFAP+ dNSC cohort (Fig. 2bii-iii). The control mice (GFAPCRE+/tk-) generated EFH neurospheres, and a subset were YFP+ (Day $24=16 \pm 2 \%$, Day $40=55 \pm 2 \%$ of all of the neurospheres formed) (Fig. 2bii,iii). Most importantly, we never observed YFP+, LIF responsive colonies in AraC+ GCV treated experimental or 

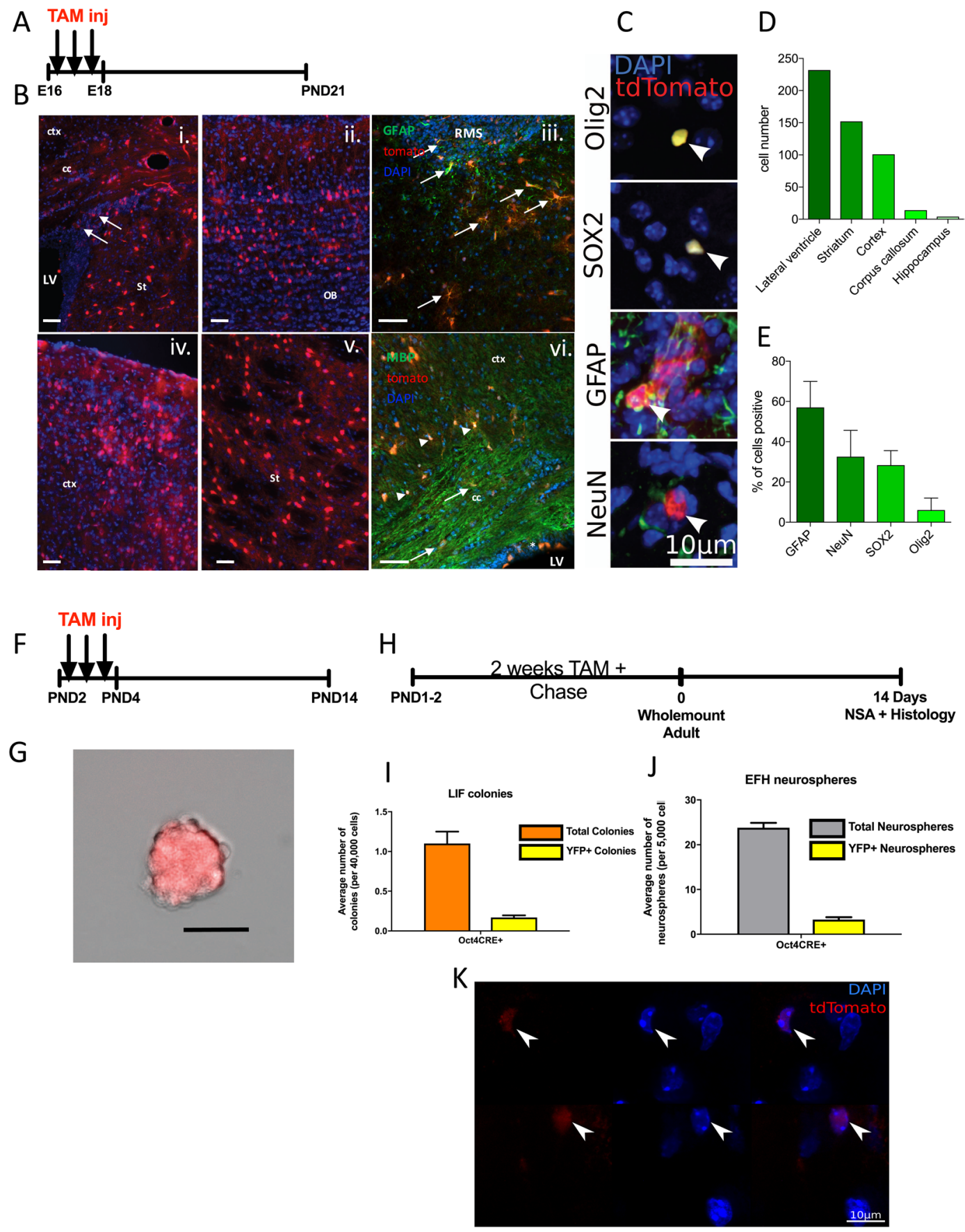

Figure 1. Identification of pNSCs in the developing and adult forebrain. (a) Schematic of the experimental paradigm for labeling Oct4 + pNSCs during embryogenesis (E16-18). (b) Sections showing tdTomato+ cells (red) throughout the brain of PND21 mice in (i) the germinal zone adjacent to the lateral ventricles (LV), (ii) olfactory bulb (OB), (iii) rostral migratory stream (RMS) with adjacent cells co-localized with GFAP (green; arrows), (iv) the cortex (ctx), (v) the striatum (St), and (vi) the corpus callosum (cc, arrow) and cortex (ctx, arrowhead) co-localized with the marker for MBP (green). Scale bars $=50 \mu \mathrm{m}$. blue $=$ Hoescht. (c) High magnification, confocal images of all lineages identified at PND21 that co-localize with tdTomato+, pNSC derived cells including oligodendrocytes (Olig2), undifferentiated progenitors (Sox2), astrocytes (GFAP), and neurons (NeuN). (d) Regionalization of tdTomato + cells throughout the brain. (e) Relative percentage of various cell types within the tdTomato + cells located in the PND21 brains. (f) Schematic of experimental paradigm for labeling Oct4 + pNSCs during early post-natal period (PND2-4). (g) Representative image of a TdTomato + LIF colony at PND14 following early post-natal labeling. Scale bar $=50 \mu \mathrm{M}$. (h) Schematic of experimental paradigm for labeling pNSCs postnatally. (i) At 4 weeks post-TAM the numbers of pNSCs (LIF) (orange bars) and co-expressing YFP + neurospheres is indicated in yellow bars ( $\mathrm{n}=5$ mice/group). (j), At 4 weeks post-TAM the total numbers of dNSCs (grey bars) and co-expressing YFP + neurospheres (yellow bars) was assessed ( $\mathrm{n}=5$ mice/group). (k) Wholemount of the lateral ventricles at 2 weeks post-TAM induction. Arrowheads indicate Tdtomato + cells. Blue $=$ DAPI, Red $=$ Tdtomato. All data represents mean \pm SEM. 
A

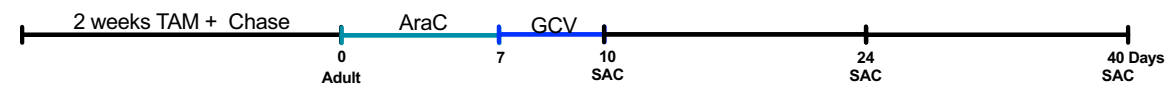

B

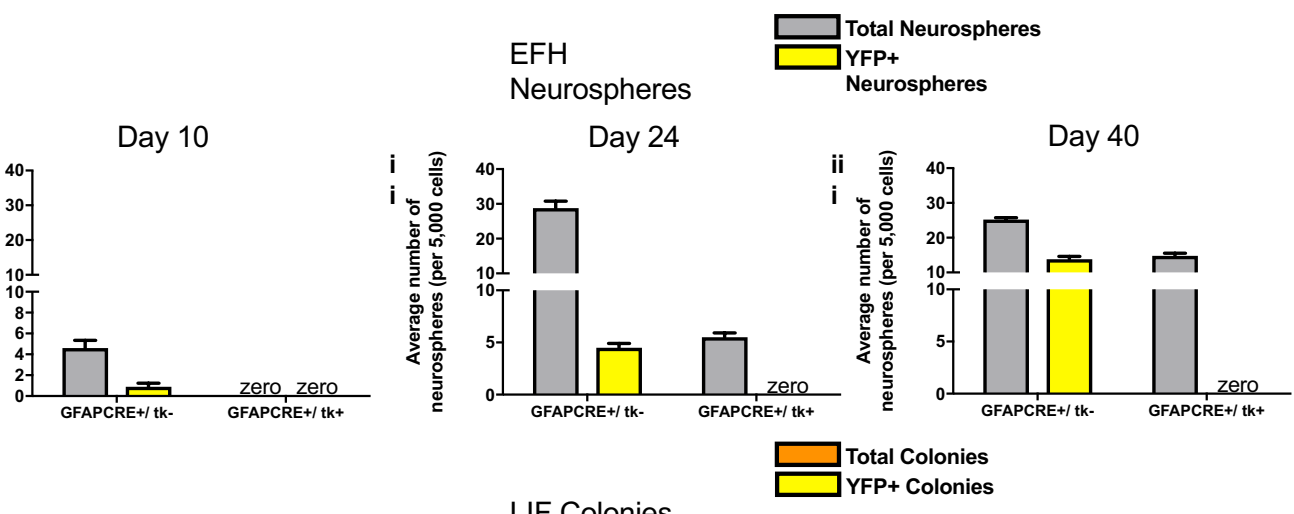

C

LIF Colonies

Day 10

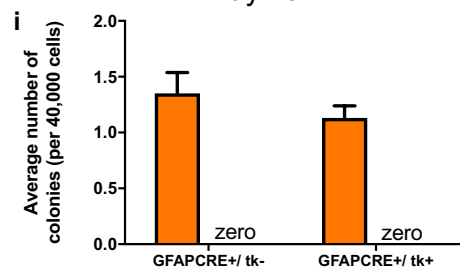

Day 24

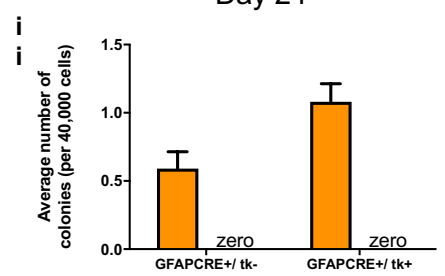

Day 40

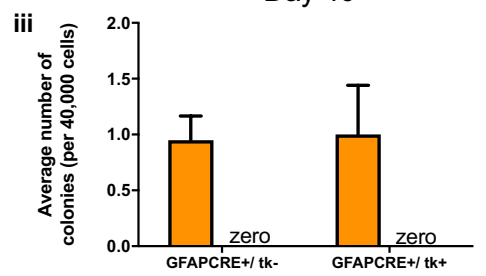

Figure 2. Repopulation of dNSCs from a non-GFAP expressing cell. (a) Schematic of the experimental paradigm. (b) The neurosphere assay for dNSCs (EFH) (grey bars) performed in control (GFAPCRE $+/$ tk - ) and experimental groups (GFAPCRE $+/ \mathrm{tk}+$ ) at day 10 (i), day 24 (ii), or day 40 (iii) after the onset of ablation. The numbers of YFP + neurospheres are indicated in yellow bars $(\mathrm{n}=6 \mathrm{mice} / \mathrm{group} / \mathrm{survival}$ time). (c) The colony-forming assay for pNSCs (LIF) (orange bars) performed in control (GFAPCRE $+/ \mathrm{tk}-$ ) and experimental groups (GFAPCRE +/tk+) at day 10 (i), day 24 (ii), or day 40 (iii) after onset of ablation. The numbers of YFP+ colonies are indicated in yellow bars $(n=6$ mice/group/survival time). All data represent mean $\pm S E M$.

control mice, at any time examined, confirming their lack of GFAP expression (Fig. 2ci-iii). The number of pNSC derived clonal colonies was not significantly different between groups (Fig. 2ci-iii; two-way ANOVA, $\mathrm{p}>0.05$ ).

Furthermore, taking advantage of a GFAP reporter mouse, we performed a related but distinct ablation to examine the precision of ablating the dNSC population. GFAP- gfp mice received intraventricular infusion of $2 \% \mathrm{AraC}$ for 7 days and immediately sacrificed (Suppl. Fig. 1a). Neurosphere formation from dNSCs in vehicle infused mice were normal; however, in AraC ablated mice, there were no EFH neurospheres, demonstrating an effective elimination of dNSCs (Suppl. Fig. 1b). Not surprisingly, the more quiescent pNSC population was unaffected by the ablation, and LIF colony formation remained at control levels and never expressed GFAP at discernible levels (Suppl. Fig. 1c). In vivo sections of these brains reveal that following AraC ablation, there is a loss of GFAP expressing cells in the subependyma of GFAP-gfp mice (Supp. Fig. 1d) not seen in vehicle infused controls (Suppl. Fig. 1e), further confirming the ablation of the dNSC population and their progeny in this model. Taken together, these findings demonstrate that repopulation of the dNSC pool following ablation occurs from a GFAP negative cell.

Ablation of dNSCs leads to the proliferation of a cohort of pNSCs that contribute to dNSC repopulation. To demonstrate that a subset of pNSCs are recruited to contribute to the repopulation of the dNSC pool following ablation, a series of experiments were carried out that relied on the proliferative status of the recruited cells during repopulation. We performed retroviral labeling in adult ROSAyfp ${ }^{\mathrm{t} / \mathrm{f} f}$;GFAPtk mice that underwent the AraC $+\mathrm{GCV}$ ablation. We injected a Cre-Recombinase expressing retrovirus (RV-Cre) intraventricularly to label proliferating cells during the repopulation phase (Fig. 3a). We predicted that the GFAP negative, pNSCs cells would be recruited and actively proliferate during the repopulation phase, and thus would be labeled with the RV-Cre and express YFP + .

Immediately following the AraC + GCV ablation (day 10), the GFAPtk + mice had a complete loss of dNSC-derived neurospheres (Fig. 3bi), as previously shown (Fig. 2). As predicted, control (GFAPtk-) mice had a cohort of dNSCs that were YFP + (16.93 $\pm 1.72 \%)$, indicating that dNSCs were proliferating post-AraC infusion and were labeled with the RV-Cre (Fig. 3bi). Most interesting, YFP+ pNSC-derived LIF colonies were observed immediately following ablation (day 10) in both GFAPtk + and GFAPtk- mice, indicating that pNSCs were actively proliferating and infected with RV-Cre during the GCV infusion (Fig. 3ci). At longer survival times post-ablation (days 14 and 30), the dNSC-derived, EFH responsive neurospheres returned in both groups and 
A

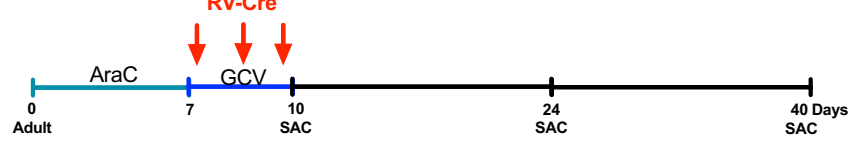

B

EFH Neurospheres $\square$ Total Neurospheres

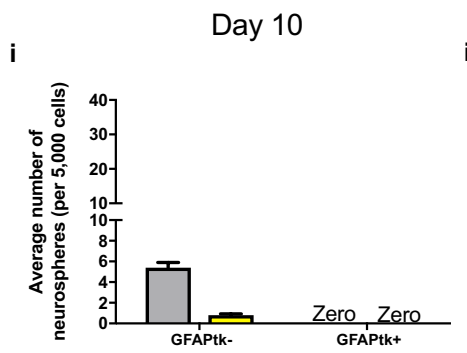

ii

Day 24

iii

Day 40

C
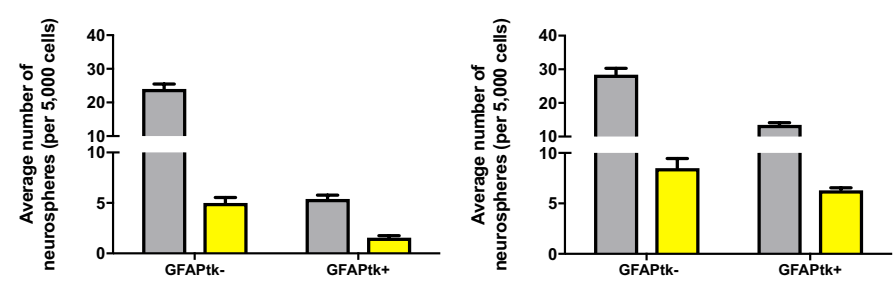

LIF Colonies

Total Colonies

YFP+ Colonies

Day 10

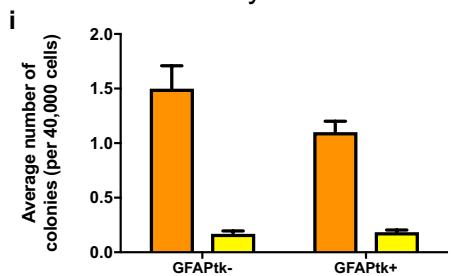

Day 24

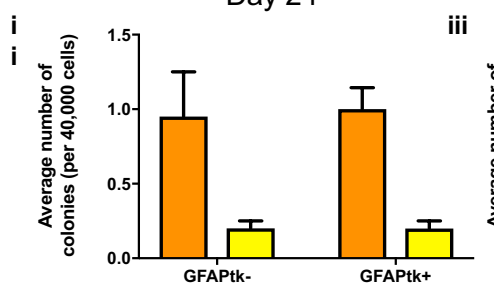

Day 40

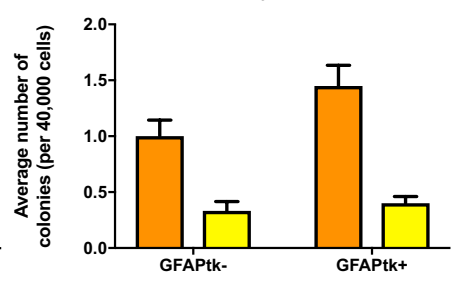

Figure 3. Proliferating, GFAP negative cells give rise to dNSCs following ablation. (a) Schematic of the experimental paradigm. (b) The neurosphere assay for dNSCs (EFH) (grey bars) performed in control (GFAPtk-) and experimental groups (GFAPtk+) at day 10 (i), day 24 (ii), or day 40 (iii) after onset of ablation. The numbers of YFP + neurospheres are indicated in yellow bars ( $\mathrm{n}=6 \mathrm{mice} / \mathrm{group} / \mathrm{survival}$ time). (c) Colonyforming assay for pNSCs (LIF) (orange bars) performed in control (GFAPtk-) and experimental groups $($ GFAPtk + ) at day 10 (i), day 24 (ii), or day 40 (iii) after the onset of ablation. The numbers of YFP+ colonies are indicated in yellow ( $n=6$ mice/group for day $10 ; n=3$ mice/group for day $24 ; n=4-6$ mice/group for day 40$)$. All data represent mean \pm SEM.

most importantly, a subpopulation of dNSC-derived neurospheres in GFAPtk+ mice were YFP+ (Fig. 3bii-iii). Thus, the repopulating cell in the experimental group is a GFAP-, proliferating pNSC cell, labeled by the RV-Cre during the GCV infusion (Fig. 3bi-iii).

The GFAPtk- control mice also underwent rapid repopulation of dNSC derived neurospheres, and a subpopulation of the dNSCs are YFP + (Day $24=20.71 \pm 1.73 \%$, Day $40=29.77 \pm 2.39 \%$ of all neurospheres). Notably, absolute numbers and the percentage of YFP + pNSC derived, LIF colonies remained constant at all times examined and in both experimental and control groups (Fig. 3ci-iii) suggesting that pNSCs undergo asymmetric divisions in vivo. Together, these findings indicate that pNSCs proliferate in response to dNSC depletion and may serve as precursors for dNSCs.

Oct4 + pNSCs are responsible for the repopulation of dNSCs. Finally, we generated triple transgenic Oct4CreER ${ }^{\mathrm{T} 2} ; \mathrm{ROSAyfp}^{\mathrm{f} / \mathrm{fl}}$;GFAPtk mice (herein called (Oct4CRE+/tk) that permit the labeling of Oct 4 expressing pNSCs with TAM induction, as well as the selective ablation of dNSCs using AraC + GCV, to examine the in vivo repopulation of dNSCs. Neonatal mice received TAM labeling from PND1-14 followed by a 4 week chase. Mice sacrificed at 6 weeks of age, prior to AraC + GCV treatment, had 4.2-7.4\% of pNSC-derived colonies expressing YFP + in both control (Oct4CRE +/tk-) and experimental (Oct4CRE +/tk+) groups. Young adult mice received $\mathrm{AraC}+\mathrm{GCV}$ treatment and were sacrificed immediately following ablation or 30 days later (day 10 and day 40, respectively). YFP+, pNSC-derived colonies were seen in control ( $9 \%$ on day $10 ; 16 \%$ on day 30 ) and experimental mice ( $14.89 \%$ on day $10 ; 15.31 \%$ on day 40 ) (Fig. 4 bi-iii). As predicted, dNSCs were completely ablated in the $\mathrm{CRE}+/ \mathrm{tk}+$ group (Fig. 4ci). Most interesting, YFP + dNSC-derived neurospheres were seen at day 40 in CRE $+/ \mathrm{tk}+$ mice revealing their derivation from Oct4 + pNSCs labeled prior to ablation (Fig. 4cii). These in vivo labeling studies reveal that Oct $4+$ pNSCs can give rise to GFAP $+\mathrm{dNSCs}$.

To further confirm the lineage relationship between pNSCs and their downstream progeny, we used Oct4CreERT2;tdTomatofl mice and subjected them to the 7 days AraC ablation paradigm (Suppl. Fig. 1). In a first series of experiments, Oct4CreERT2;tdTomatofl mice were fed tamoxifen through their mother's milk for PND014 and allowed to chase until young adults (Fig. 5a). Neurosphere assays performed following this recombination paradigm demonstrated that only a rare population of pNSCs are labeled in these mice using tamoxifen-induced recombination (Fig. 5b,c). In Oct4CreERT2;tdTomatofl mice that received ablation, despite the rarity of pNSCs 
A

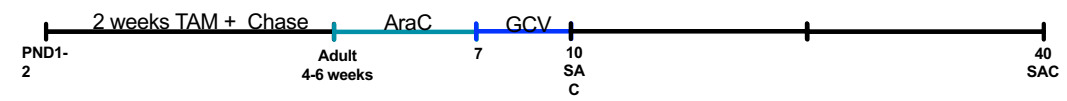

B LIF Colonies $\quad \square$ Total Colonies

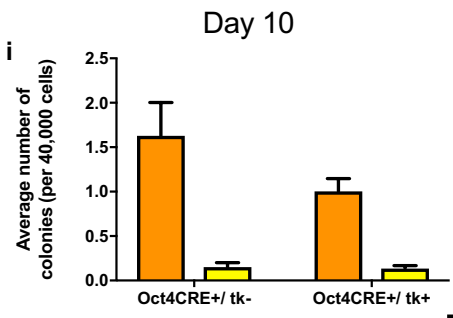

C

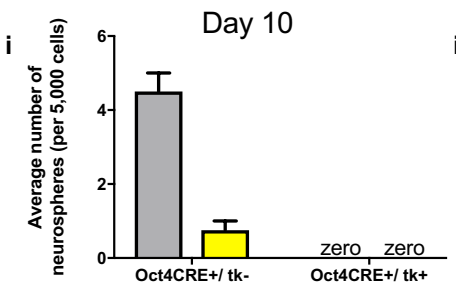

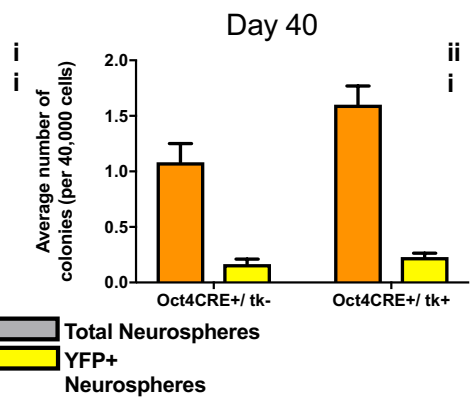

Day 40
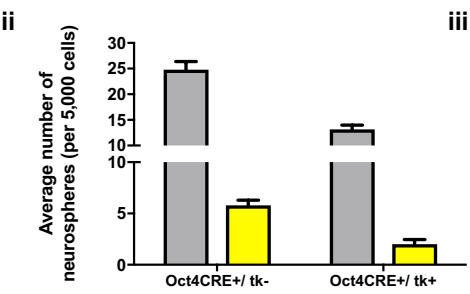

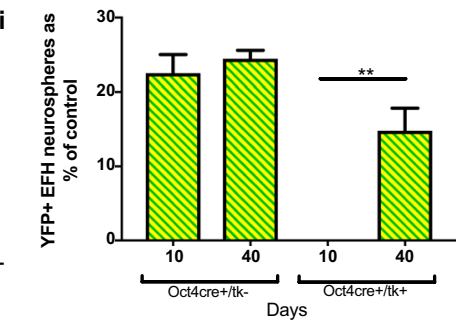

Figure 4. Oct 4 expressing, primitive NSCs give rise to dNSCs following ablation. (a) Schematic of the experimental paradigm. (b) The colony-forming assay for pNSCs (LIF) (orange bars) was performed in control $(\mathrm{Oct} 4 \mathrm{CRE}+/ \mathrm{tk}-)$ and experimental mice $(\mathrm{Oct} 4 \mathrm{CRE}+/ \mathrm{tk}+)$ at day 10 (i) or day 40 (ii). The numbers of YFP+ colonies are indicated in yellow bars. Day $10, \mathrm{n}=3$ Oct $4 \mathrm{CRE} / \mathrm{tk}-$ mice, $\mathrm{n}=5$ Oct $4 \mathrm{CRE} / \mathrm{tk}+$ mice; Day 40 , $\mathrm{n}=5$ mice/group. (c) The numbers of dNSCs (EFH) (grey bars) in control (Oct4CRE+/tk - ) and experimental groups (Oct4CRE $+/ \mathrm{tk}+$ ) are assessed at day 10 (i) or day 40 (ii). The numbers of YFP + neurospheres are indicated in yellow bars. Day $10, \mathrm{n}=3$ mice/group for day 10 ; Day $40, \mathrm{n}=3$ Oct4CRE/tk- mice, $\mathrm{n}=5$ Oct4CRE/ $\mathrm{tk}+$ mice. All data represent mean \pm SEM.

in adulthood, following tamoxifen food (Fig. 5d) we found tdTomato+ cells in the subependyma (Fig. 5e). Most interesting, we also observed mature neurons in the olfactory bulb expressing tdTomato, indicating they were derived from pNSCs (Fig. 5f). pNSCs may give rise to more differentiated progeny directly without going through a dNSC state in addition to giving rise to dNSCs (Fig. 5g). Excitingly, this confirms that pNSCs give rise to all cells in the neural lineage and reside at the top of the neural stem cell hierarchy.

\section{Discussion}

Lineage tracing has demonstrated the in vivo relationship between the two NSC populations found in the mouse forebrain. Using several transgenic mouse models that permit the selective labeling of dNSCs and pNSCs in the forebrain subependyma, and the selective ablation of dNSCs, we demonstrate that pNSCs are upstream of the dNSC population. This lineage relationship was demonstrated in naïve mice as well as after dNSC ablation.

Under baseline conditions, we have shown that pNSCs generate cells of all three lineages during embryogenesis, and that the progeny are widespread throughout the brain parenchyma. Interestingly, we found that the majority of pNSC progeny are neuronal in the early post-natal brain. This suggests that pNSCs from E16-18 are more likely to expand symmetrically and generate neuronal progeny than give rise to dNSCs or glial progenitors during this time. The fact that we observed precursors in the periventricular region of the PND21 brain suggests that the pNSCs generated progeny that became quiescent in vivo. Importantly, the dNSCs that arise from pNSCs are maintained into adulthood. Understanding the direct connection between pNSCs and dNSCs and their capacity to generate all neural cell types is key in positing predictions about their behavior post-ablation and their potential to contribute to neural repair post-injury. Recent findings have identified pNSCs along the entire neuraxis of the developing and adult mouse CNS, and further, spinal cord injury models have demonstrated that pNSCs can migrate to the site of injury in the injured spinal cord ${ }^{13}$. This raises the possibility that pNSCs can respond to migratory cues and could contribute to neural repair.

The lack of increase in the size of the pNSC pool suggests that pNSCs divide asymmetrically to generate dNSCs during repopulation. While we have shown that pNSCs can give rise to $\mathrm{dNSCs}$, it remains possible that pNSCs give rise directly to neuronal progeny without going through a dNSC state. Indeed, the fact that pNSCs transplanted onto the rostral migratory stream give rise to neuroblasts and neurons in the olfactory bulb supports this lineage relationship $^{7}$. Further, we cannot rule out the possibility that a more quiescent subpopulation of dNSCs also contributes to 
A

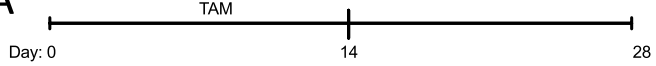

B

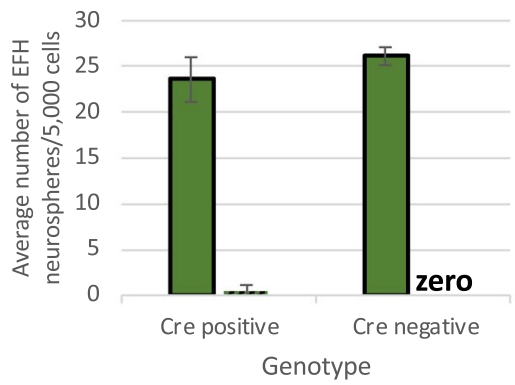

C
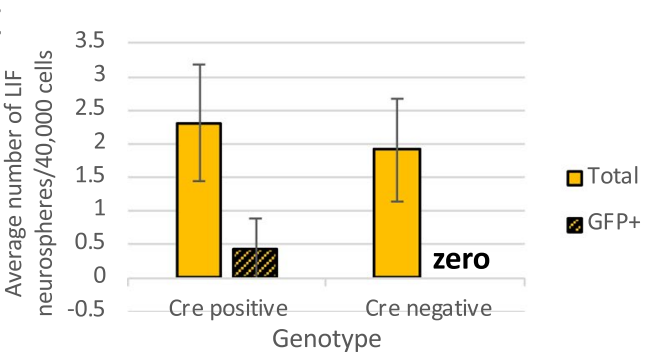

D Day: 0

AraC

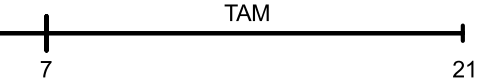

E

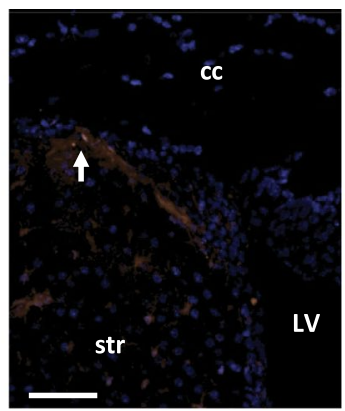

F

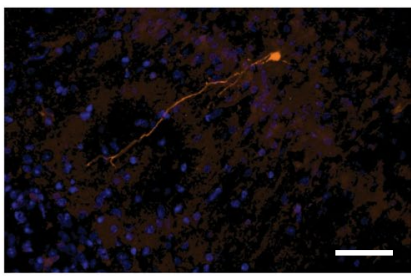

G

Olfactory Bulb

G

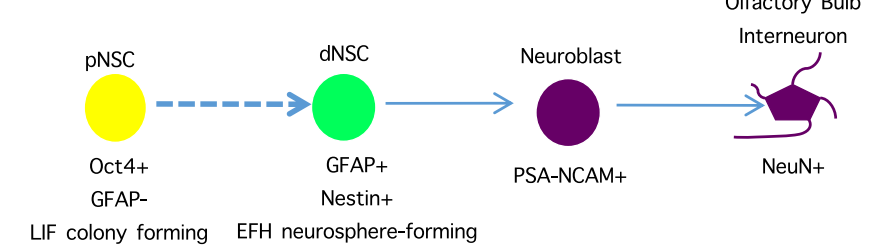

Figure 5. Oct4 expressing, primitive NSC contribution to the neural lineage. (a) Schematic of the experimental paradigm. (b) Neurosphere assay performed from Oct4CreERT2;tdTomatofl mice that had been fed tamoxifen assayed for dNSCs derived from pNSCs (Cre positive) against controls (Cre negative). (c) The number of pNSC derived colonies labeled assessed in Oct4CreERT2;tdTomatofl mice (Cre positive) versus controls (Cre negative). (d) Schematic of the experimental paradigm. (e) Rare tdTomato + , Oct4 expressing cells are located in the dorsolateral corner of the lateral ventricles during repopulation following ablation with AraC of the dNSCs and progeny. Scalebar $=100 \mu \mathrm{m} . \mathrm{LV}=$ lateral ventricle, $\mathrm{str}=$ striatum, $\mathrm{cc}=$ corpus callosum. $(\mathbf{f})$ tdTomato + neurons located in the olfactory bulb derived from Oct4 expressing pNSCs. Scalebar $=100 \mu \mathrm{m}$. (g) Proposed lineage where dNSC in the adult brain SVZ are GFAP expressing cells that form clonally derived neurospheres in the presence of growth factors (EGF and FGF and heparin)(EFH). These dNSC proliferate to give rise to migrating neuroblasts on the rostral migratory stream. Neuroblasts migrate to the olfactory bulb where they differentiate into olfactory bulb interneurons. Rare GFAP negative, Oct 4 positive, LIF responsive, colony-forming cell are found in the periventricular region of the adult brain. These pNSCs are found during development, as early as E5.5, and persist into adulthood.

the subependymal repopulation post-ablation ${ }^{14}$, and this would be consistent with the observation that differential levels of GFAP protein expression in dNSCs has been demonstrated in vivo ${ }^{15}$. Notably, while differential GFAP expression could explain why no YFP expressing dNSC-derived neurospheres were seen following ablation (i.e. if the GFAP expression was too low to be depleted by the GCV), it cannot explain the current observation that Oct4+ (YFP expressing) cells give rise to dNSCs in vivo. Hence, while our work demonstrates that pNSCs give rise to dNSCS, we cannot rule out the possibility that pNSCs can bypass the dNSC state and give rise directly to lineage-committed cells during development or in the adult during SVZ repopulation.

Recent work has demonstrated that pNSCs and dNSCs are differentially regulated populations of neural stem cells that exist along the entire neuraxis; in both neurogenic (forebrain) and aneurogenic regions (spinal cord) ${ }^{9,13}$. Further it has been suggested that the dNSC population present in the adult is derived from a developmental population and set aside early in development ${ }^{16}$, which is consistent with the idea that pNSCs are most abundant

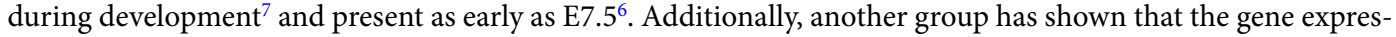
sion of the dNSC population exists on a continuum throughout development and adulthood ${ }^{17}$, which falls in line with our data demonstrating pNSCs persisting into adulthood. We propose that pNSCs give rise to dNSCs during development and persist as a reserve source of stem cells in the mature CNS, which can be activated in response to injury ${ }^{4,7}$. 


\begin{abstract}
Methods
Mice. Mice were housed within the Department of Comparative Medicine at the University of Toronto. The University Animal Care Committee (UACC) and Research Oversight and Compliance Office at the University of Toronto approved the research ethics, in accordance with the guidelines of the Canadian Council of Animal Care prior to experimentation. The following transgenic mice, both sexes, were used in this study. GFAP-TK Line 7.1, a kind gift from Dr. M Sofroniew (UCLA), bred in house. GFAP-CreER ${ }^{\mathrm{T} 2}$ and GFAP-gfp, a kind gift from Dr. A Nagy (University of Toronto), bred in house. Rosa26-EYFPfl (http://jaxmice.jax.org/strain/006148. html), Pou5f1 tm1.1(Cre/Esr1)Yseg (https://www.jax.org/strain/016829) and B6;129S6-Gt(ROSA)26Sor ${ }^{\text {tm9(CAG-tdTomato) }}$ Hze/J (https://www.jax.org/strain/007905) purchased from The Jackson Laboratories (USA). Combinations

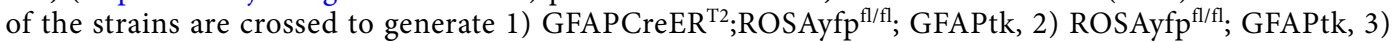

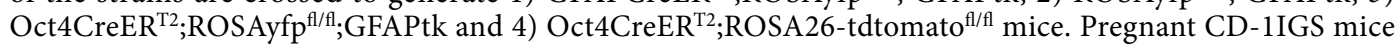
(http://www.criver.com/products-services/basic-research/find-a-model/cd-1-mouse) (Charles River (Canada)) were used for fostering.
\end{abstract}

Dissection and in vitro assays. The neurosphere assay was performed as previously described ${ }^{18}$. Briefly, mice were killed with an overdose of avertin or isoflurane, and the brain was removed. The periventricular region lining the lateral ventricle was dissected and dissociated into single cells using enzymatic treatment (trypsin $(1.33 \mathrm{mg} / \mathrm{ml})$ and hyaluronidase $(0.67 \mathrm{mg} / \mathrm{ml})$ and kynurenic acid $(0.2 \mathrm{mg} / \mathrm{ml})$ - Sigma-Aldrich, St. Louis, MO) and mechanical trituration. Cells are plated at clonal density ( 10 cells/ul) (a density at which spheres are derived from single starting cell $\left.\mathrm{s}^{19}\right)$ in serum free media containing l-glutamine ( $2 \mathrm{mM}$, Invitrogen), penicillin/streptavi$\operatorname{din}(100 \mathrm{U} / 0.1 \mathrm{mg} / \mathrm{ml}$, Invitrogen), mitogens (epidermal growth factor (EGF, $20 \mathrm{ng} / \mathrm{ml}$ ); fibroblast growth factor (FGF, $10 \mathrm{ng} / \mathrm{ml}$ ); heparin ( $2 \mathrm{ug} / \mathrm{ml}$ ), Sigma) for dNSCs; or leukemia inhibitory factor (LIF, $10 \mathrm{ng} / \mathrm{ml}$ ) for pNSCs). EFH is used to denote the dNSC media conditions. Primary neurospheres were counted after 7 days with the following strict inclusion criteria: pNSCs $>50 \mu \mathrm{m}$ and dNSCs $>80 \mu \mathrm{m}$ in diameter.

Tamoxifen labelling. To label the Oct4+ cells during development timed pregnant female Oct4CreER ${ }^{\mathrm{T} 2}$;ROSA26-Tdtomato ${ }^{\mathrm{f} / \mathrm{fl}}$ were injected with tamoxifen (Sigma) on embryonic day 16 and once daily for 3 days (E16-18). Tamoxifen was prepared as previously described ${ }^{20}$ in a suspension of $10 \%$ ethanol in $90 \%$ sunflower oil at a concentration of $30 \mathrm{mg} / \mathrm{ml}$. Each mouse received an intraperitoneal injection of $3 \mathrm{mg} /$ day taking care to avoid the developing fetuses. Fetuses were delivered by cesarian section, fostered to CD1 lactating dams and allowed to survive for 3 weeks.

For labeling cells in adult animals, young 4 week-old GFAPCreER ${ }^{\mathrm{T} 2} ; \mathrm{ROSAyfp}^{\mathrm{fl} / \mathrm{fl}} ; \mathrm{GFAPtk}$ mice were fed tamoxifen chow $\left(250 \mathrm{mg} / \mathrm{kg}\right.$, Envigo) for 2 weeks followed by a 2 -week chase. For Oct4CreER ${ }^{\mathrm{T} 2} ; \mathrm{ROSAyfp}^{\mathrm{f} / \mathrm{fl}}$ and Oct4CreER ${ }^{\mathrm{T} 2}$;ROSA26-Tdtomato ${ }^{\mathrm{fl} / \mathrm{fl}}$ mice, post-natal day (PND) 1 pups are fostered to CD-1 mothers that had age matched litters. CD-1 mothers were fed tamoxifen chow $(250 \mathrm{mg} / \mathrm{kg}$, Envigo) for 2 weeks, followed by a 4 week chase.

Ablation paradigms. Mice were anaesthetized with $5 \%$ isoflurane (inhalation) and ketoprofen $(5 \mathrm{mg} / \mathrm{kg}$; injected SQ). Alzet 1007D mini osmotic pumps ( $0.05 \mathrm{ul} /$ hour, Direct Corp.) containing $2 \%$ L-arabinose cytarabine (AraC) were implanted subcutaneously and attached to a cannula implanted into the lateral ventricle at the coordinates: $0.2 \mathrm{~mm}$ AP from bregma, $0.7 \mathrm{~mm} \mathrm{ML}$, and $2.5 \mathrm{~mm}$ DV below the dura. AraC was delivered for 7 days followed immediately by sacrifice, tamoxifen chow for two weeks, or infusion of ganciclovir (GCV) (200uM, Sigma) for 3 days.

Injection cannulation and retrovirus injection. $\quad$ ROSAyf $\mathrm{p}^{\mathrm{fl} / \mathrm{fl}} ; \mathrm{GFAPtk}$ mice were anaesthetized with $5 \%$ isoflurane (inhalation) and ketoprofen ( $5 \mathrm{mg} / \mathrm{kg}$; injected SQ). Injector cannula (C315G2-2-SP Guide, Plastics One) was placed in the lateral ventricle of the opposite hemisphere to the mini osmotic pumps and secured to the surface of the skull with dental cement. Retrovirus carrying Cre-Recombinase (in-house ${ }^{21}$ ) was injected through the cannula daily at $3 \mathrm{uL}$ for 3 days during GCV infusion.

Tissue preparation and immunohistochemistry. Wholemount sections derived from Oct4CreER ${ }^{\mathrm{T} 2}$;ROSA26-tdtomato ${ }^{\mathrm{f} / \mathrm{fl}}$ mice as previously described ${ }^{7,22}$. Briefly, animals were overdosed as described and perfused with PBS. Brains were removed and the walls of the lateral ventricles were exposed. Wholemounts were fixed for 48 hours in 4\% PFA followed by 48 hours in 1x PBS for washing prior to incubation with Hoescht (Sigma B2261, 1:1000) in PBS for 24 hours. Wholemounts of the entire lateral wall of the ventricle were removed and mounted on superfrost glass slides, coverslipped and allowed to settle for 48 hours before visualization.

Mice that received tamoxifen injections in utero were perfused on PND21 as previously described ${ }^{7}$. Briefly, animals were overdosed with tri-bromo-ethanol (Avertin, Sigma) and transcardially perfused with ice cold PBS followed by $4 \%$ paraformaldehyde. Brains were removed and post-fixed for 4 hours in $4 \%$ paraformaldehyde and subsequently transferred to $20 \%$ sucrose in PBS for cryoprotection. Brains were frozen and serially sectioned on a cryostat to derive $30 \mu \mathrm{m}$ sections on superfrost slides (Fisherbrand, Fisher Scientific). Sections were stained with Hoescht (1:1000, Sigma) in PBS for 20 minutes to visualize tdtomato+ cells. For immunohistochemistry, slides were rehydrated in PBS for 10 min followed by a 1 hour incubation at room temperature with $10 \%$ NGS in $0.3 \%$ Tritonx 100 in PBS. Sections were incubated overnight at $4{ }^{\circ} \mathrm{C}$ with primary antibodies in $10 \%$ NGS in PBS. Primary antibodies were GFAP (Sigma; rabbit polyclonal, 1:500; or DAKO, rabbit polyclonal, 1:2000), Olig2 (Millipore, rabbit polyclonal, 1:500), SOX2 (Abcam, rabbit polyclonal, 1:2000), NeuN (Abcam, rabbit polyclonal, 1:2000), and MBP (Abcam, rat monoclonal, 1:50). Following washing, sections were incubated with secondary antibodies Alexa Fluor 488 goat anti-rabbit IgG or goat anti-rat IgG with Hoescht (1:1000, Sigma) in 10\% NGS in PBS for one hour at room temperature. Sections were washed and mounted using Mowiol (in house) and left to 
dry at room temperature overnight. Visualization of tissue was performed on a Zeiss spinning disc confocal using Zen software for acquisition or an inverted confocal microscope with a motorized stage (Olympus FV1000). The percentage of tdTomato expressing cells that co-labeled with GFAP, Olig2, Sox2, or NeuN at PND21 was calculated from sections evenly distributed throughout the brain $(\mathrm{n}=4$ mice). In addition, the location of tdTomato + cells was recorded $(\mathrm{n}=4$ mice) .

Statistics. Data are represented as mean \pm SEM. Two-tailed t-tests were performed to compare between two groups. ANOVA was used to compare between multiple groups with Dunnet's post hoc test. Significance was considered $\mathrm{p}<0.05$. All graphs and analysis were generated from either Excel (Microsoft) or GraphPad Prism 6 (Graph Pad Software, California).

Received: 24 April 2019; Accepted: 4 November 2019;

Published online: 27 November 2019

\section{References}

1. Doetsch, F., Caillé, I., Lim, D. A., García-Verdugo, J. M. \& Alvarez-Buylla, A. Subventricular zone astrocytes are neural stem cells in the adult mammalian brain. Cell 97, 703-716 (1999).

2. Morshead, C. M., Craig, C. G. \& van der Kooy, D. In vivo clonal analysis of endogenous neural stem cell proliferation in the adult mammalian forebrain. Development 125, 2251-2261 (1998).

3. Morshead, C. M., Garcia, A. D., Sofroniew, M. V. \& van Der Kooy, D. The ablation of glial fibrillary acidic protein-positive cells from the adult central nervous system results in the loss of forebrain neural stem cells but not retinal stem cells. Eur. J. Neurosci. 18, 76-84 (2003).

4. Reeve, R. L., Yammine, S. Z., Morshead, C. M. \& van der Kooy, D. Quiescent Oct4(+) Neural Stem Cells (NSCs) Repopulate Ablated Glial Fibrillary Acidic Protein(+) NSCs in the Adult Mouse Brain. Stem Cells Dayt. Ohio 35, 2071-2082 (2017).

5. Akamatsu, W., DeVeale, B., Okano, H., Cooney, A. J. \& van der Kooy, D. Suppression of Oct4 by germ cell nuclear factor restricts pluripotency and promotes neural stem cell development in the early neural lineage. J. Neurosci. Off. J. Soc. Neurosci. 29, 2113-2124 (2009).

6. Hitoshi, S. et al. Primitive neural stem cells from the mammalian epiblast differentiate to definitive neural stem cells under the control of Notch signaling. Genes Dev. 18, 1806-1811 (2004).

7. Sachewsky, N. et al. Primitive neural stem cells in the adult mammalian brain give rise to GFAP-expressing neural stem cells. Stem. Cell Rep. 2, 810-824 (2014).

8. Reynolds, B. A. \& Weiss, S. Generation of neurons and astrocytes from isolated cells of the adult mammalian central nervous system. Science 255, 1707-1710 (1992).

9. Reeve, R. L., Yammine, S. Z., DeVeale, B. \& van der Kooy, D. Targeted activation of primitive neural stem cells in the mouse brain. Eur. J. Neurosci. 43, 1474-1485 (2016).

10. Qian, X. et al. Timing of CNS cell generation: a programmed sequence of neuron and glial cell production from isolated murine cortical stem cells. Neuron. 28(1), 69-80 (2000).

11. Barnabe-Heider, F. et al. Evidence that embryonic neurons regulate the onset of cortical gliogenesis via Cardiotrophin-1. Neuron. 48(2), 253-265 (2005).

12. Miller, F. D. \& Gauthier, A. S. Timing is everything: making neurons versus glia in the developing cortex. Neuron. 54(3), 357-369 (2007).

13. Xu, W. et al. Myelin Basic Protein Regulates Primitive and Definitive Neural Stem Cell Proliferation from the Adult Spinal Cord. Stem Cells. 35, 485-496 (2017).

14. Codega, P. et al. Prospective identification and purificantion of quiescent adult neural stem cells from their in vivo niche. Neuron. 82(3), 545-559 (2014).

15. Mich, J. K. et al. Prospective identification of functionally distinct stem cells and neurosphere-initiating cells in adult mouse forebrain. eLife 3, e02669 (2014)

16. Furutachi, S. et al. Slowly dividing neural progenitors are an embryonic origin of adult neural stem cells. Nat. Neurosci. 18(5), 657-65 (2015).

17. Yuzwa, S. A. et al. Developmental emergence of adult neural stem cells as revealed by single-cell transcriptional profiling. Cell Reports. 21, 3970-3986 (2019).

18. Chiasson, B. J., Tropepe, V., Morshead, C. M. \& van der Kooy, D. Adult mammalian forebrain ependymal and subependymal cells demonstrate proliferative potential, but only subependymal cells have neural stme cell characteristics. JNeurosci. 19(11), 4462-4471 (1999).

19. Coles-Takabe, B. L. K. et al. Don't look: growing clonal versus nonclonal neural stem cell colonies. Stem Cells Dayt. Ohio 26, 2938-2944 (2008).

20. Lagace, D. C. et al. Dynamic contribution of nestin-expressing stem cells to adult neurogenesis. JNeurosci. 27946, 12623-12629 (2007).

21. Piccin, D., Yu, F. \& Morshead, C. M. Notch signaling imparts and preserves neural stem characteristics in the adult brain. Stem Cells Dev. 22, 1541-1550 (2013).

22. Mirzadeh, Z., Doetsch, F., Sawamoto, K., Wichterle, H. \& Alvarez-Buylla, A. The subventricular zone en-face: wholemount staining and ependymal flow. J. Vis. Exp. JoVE. 2010(39), 1938 (2010).

\section{Author contributions}

Conceptualization, W.X., N.S., C.M.M., and D.V.D.K.; Methodology, W.X., N.S., and C.M.M.; Investigation, W.X., N.S. and T.F.; Writing- Original Draft, W.X. and C.M.M.; Writing- Review \& Editing, N.S., W.X., T.F., D.V.D.K. and C.M.M.; Supervision, C.M.M.; Funding Acquisition, W.X. and C.M.M.

\section{Competing interests}

The authors declare no competing interests.

\section{Additional information}

Supplementary information is available for this paper at https://doi.org/10.1038/s41598-019-54143-9.

Correspondence and requests for materials should be addressed to C.M.M.

Reprints and permissions information is available at www.nature.com/reprints. 
Publisher's note Springer Nature remains neutral with regard to jurisdictional claims in published maps and institutional affiliations.

(c) (i) Open Access This article is licensed under a Creative Commons Attribution 4.0 International License, which permits use, sharing, adaptation, distribution and reproduction in any medium or format, as long as you give appropriate credit to the original author(s) and the source, provide a link to the Creative Commons license, and indicate if changes were made. The images or other third party material in this article are included in the article's Creative Commons license, unless indicated otherwise in a credit line to the material. If material is not included in the article's Creative Commons license and your intended use is not permitted by statutory regulation or exceeds the permitted use, you will need to obtain permission directly from the copyright holder. To view a copy of this license, visit http://creativecommons.org/licenses/by/4.0/.

(c) The Author(s) 2019 